\title{
Congenitally Corrected Transposition of the Great Arteries: Conduction Anomalies: A Case Report
}

\author{
Jose Antonio Luna-Alvarez-Amezquita ${ }^{1}$, Javier Ivan Armenta-Moreno ${ }^{1}$, Joaquin Berarducci ${ }^{1}$,

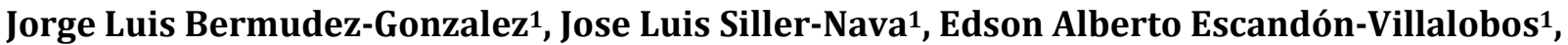 \\ Nilda Espinola-Zavaleta ${ }^{1,2}$, Roberto Cano-Zarate ${ }^{1,3}$, Juan Ignacio Straface ${ }^{1^{*}}$
}

${ }^{1}$ Department of Nuclear Cardiology, National Institute of Cardiology Ignacio Chavez, Mexico City, Mexico

${ }^{2}$ Department of Echocardiography, ABC Medical Center I.A.P., Mexico City, Mexico

${ }^{3}$ Department of Magnetic Resonance, National Institute of Cardiology Ignacio Chavez, Mexico City, Mexico

Email: *uanistraface@gmail.com

How to cite this paper: Luna-AlvarezAmezquita, J.A., Armenta-Moreno, J.I., Berarducci, J., Bermudez-Gonzalez, J.L., Siller-Nava, J.L., Escandón-Villalobos, E.A., Espinola-Zavaleta, N., Cano-Zarate, R. and Straface, J.I. (2021) Congenitally Corrected Transposition of the Great Arteries: Conduction Anomalies: A Case Report. World Journal of Cardiovascular Diseases, 11, 439-444.

https://doi.org/10.4236/wjcd.2021.119041

Received: July 28, 2021

Accepted: September 11, 2021

Published: September 14, 2021

Copyright $\odot 2021$ by author(s) and Scientific Research Publishing Inc. This work is licensed under the Creative Commons Attribution International License (CC BY 4.0).

http://creativecommons.org/licenses/by/4.0/

\begin{abstract}
Introduction: Congenitally corrected transposition of the great arteries (CCTGA) is a rare heart disease that encompasses an atrioventricular and ventriculoarterial discordance and accounts for less than 1 percent of congenital heart diseases. Objective: To present an atypical case of a man with complex congenital heart disease and conduction anomalies. Case Presentation: This is a case of a 34-year-old patient who came to the hospital with 1 week of dyspnea on exertion and episodes of lipothymia. The patient was referred to our hospital after an electrocardiogram from his primary care with evidence of blocked atrial fibrillation. During the initial evaluation blocked atrial fibrillation was confirmed with a rescue ventricular rate of $38 \mathrm{bpm}$. A magnetic resonance confirmed the presence of the atrioventricular and ventriculoarterial discordance, the aorta had a left anterior position, perimembranous ventricular septal defect with a right to left shunt, biventricular systolic dysfunction, moderate tricuspid, and mitral regurgitation, interventricular septal intramyocardial and biatrial fibrosis, left atrial dilation, and dilation of the pulmonary artery. After pacemaker placement, the patient has an improvement in his clinical symptoms and quality of life. Conclusions: Cardiac arrhythmias are CCTGA's leading cause of death, mostly ventricular tachycardia, and atrial fibrillation. Right bundle branch block is a previously unreported and potentially very rare presentation of this disease. This, added to the fact that our patient was diagnosed at an advanced age, but without symptoms of heart failure, makes him an atypical case of CCTGA, with new potential treatment options.
\end{abstract}


Keywords

Congenital Heart Disease, Congenitally Corrected Transposition of the Great Arteries, Cardiac Magnetic Resonance, Echocardiography, Arrhythmia

\section{Introduction}

Congenitally corrected transposition of the great arteries (CCTGA) is a rare heart disease that encompasses an atrioventricular and ventriculoarterial discordance. This accounts for less than 1 percent of all congenital heart diseases. The disease is usually associated with other cardiac anomalies, such as ventricular septal defects, pulmonary stenosis, tricuspid and mitral valve lesions, and others [1].

Most of the patients with CCTGA are asymptomatic until adulthood, where the most common clinical presentation is due to heart failure exacerbations. It has been hypothesized that the central cause which leads to heart failure is the incompetence of the right ventricle to maintain the systemic circulation through a person's life [1] [2].

Conduction abnormalities are another common cause of decompensation [3]. The most common arrhythmia in CCTGA is auriculoventricular heart block, followed by supraventricular tachyarrhythmias, mainly atrial fibrillation, and atrial flutter. The presence of right and left bundle branch blocks and ventricular arrhythmias are usually found in patients with heart failure and is thought to be a complication rather than part of CCTGA [1] [4].

The diagnosis of CCTGA is complex and it is based on multiple imaging modalities. The most important image studies to establish CCTGA are magnetic resonance imaging (MRI) and a comprehensive echocardiogram, paying special attention to the anatomical details of the right and left ventricles, as well as both atria. The crux of the heart is another anatomical reference that should be evaluated to differentiate CCTGA from the criss-cross heart. Finally, the position of the aorta and pulmonary artery should be assessed to record the parallel presentation of both structures and make the final diagnosis [5].

Treatment for CCTGA is based on the management of heart failure symptoms and the prevention and correction of arrhythmias using a pacemaker. Patients that undergo surgical correction procedures have an improvement in clinical symptoms and quality of life, without observing a significant change in survival time. Patients who get into adult life without a surgical procedure develop heart failure earlier compared to those who had the anatomical correction. It is also recommended to give an implantable defibrillator to patients with heart failure with severely reduced ejection fraction [2] [6].

\section{History of Presentation}

We present the case of a 34-year-old patient who came to the hospital with 1 
week of dyspnea on exertion and episodes of lipothymia. The patient was referred to our hospital after an electrocardiogram from his primary care with evidence of blocked atrial fibrillation. During the initial evaluation blocked atrial fibrillation was confirmed with a rescue ventricular rate of $38 \mathrm{bpm}$. Analyzing the QRS complex, it had a duration of $110 \mathrm{~ms}$ from V1 to V3, and an rSR' pattern in the same leads, deep $S$ waves with a duration of $50 \mathrm{~ms}$ in leads D1 and V6, compatible with diagnosis of incomplete right bundle branch block (Figure 1).

No relevant family history was obtained. Smoking since age 12 (11 packages per year). A heart murmur was detected at 4 years old, which was treated in his city until the patient turned 16 years old, when he stopped coming to medical follow-ups, however, past medical records are not available. Patient reports having reduced exercise capacity compared to his peers since childhood.

Due to a heart murmur detected on evaluation and his past medical history, a transthoracic echocardiogram was performed, which showed CCTGA and a perimembranous ventricular septal defect. Posteriorly, an MRI confirmed the presence of the atrioventricular discordance (Figure 2(a)), ventriculoaterial discordance (Figure 2(b) \& Figure 2(c)) with aorta in left anterior position, perimembranous ventricular septal defect with a right to left shunt, biventricular systolic dysfunction, moderate tricuspid and mitral regurgitation (Figure 3), left atrial enlargement and hyperintensity in both atria and in the intramyocardial ventricular septum (Figure 3).

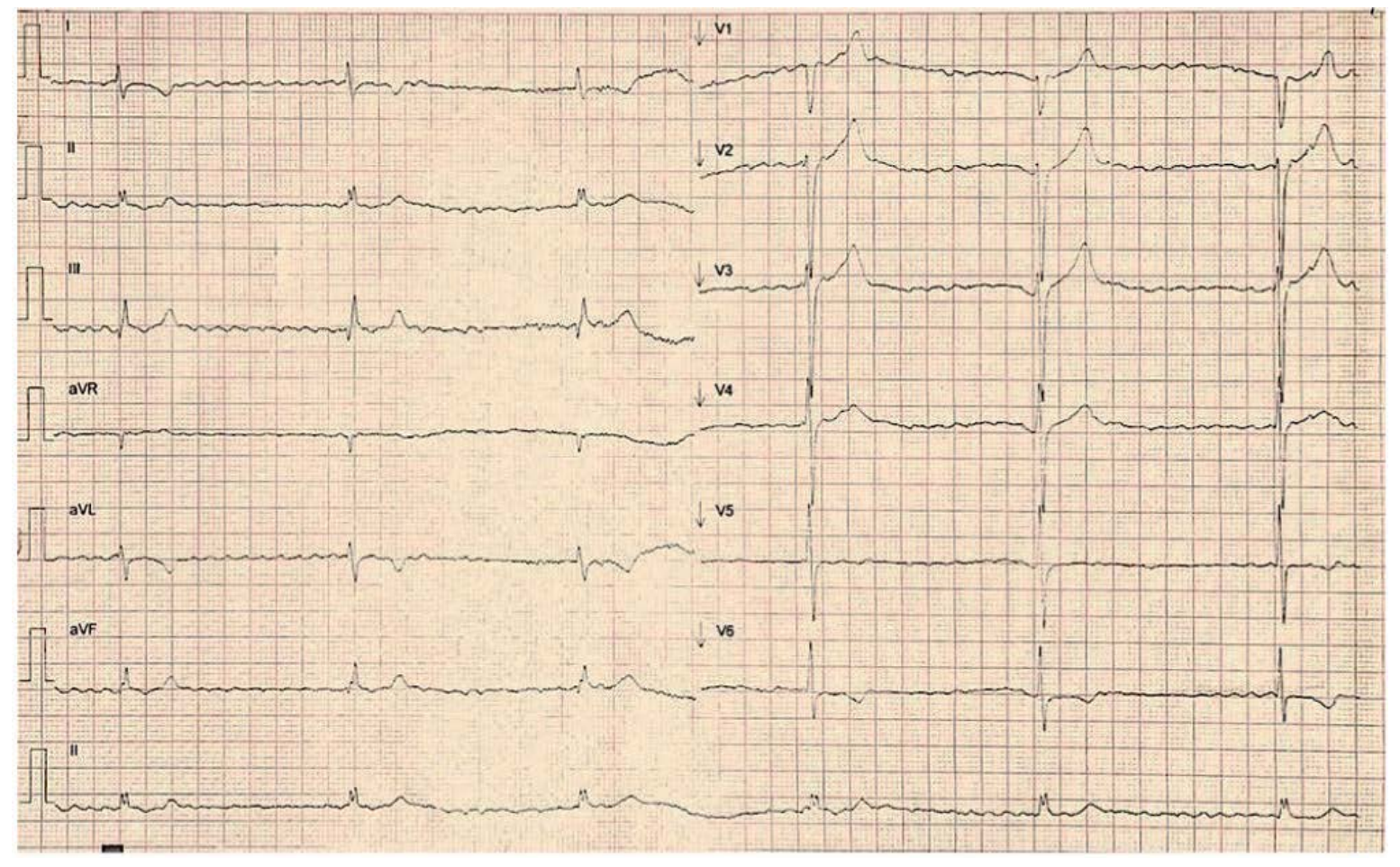

Figure 1. ECG. Blocked atrial fibrillation with a mean heart rate of 36 beats/min, rSR' pattern in the V1 toV3 leads, compatible with incomplete right bundle branch block. 


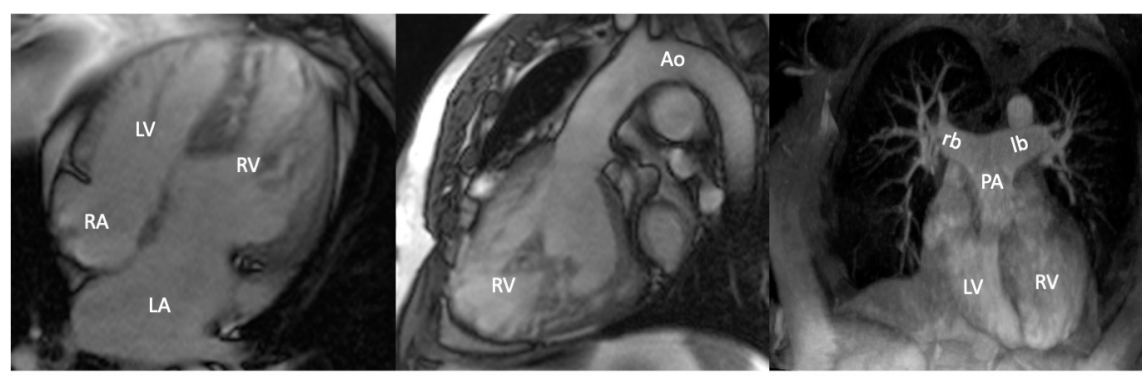

(a)

(b)

(c)

Figure 2. (a) Four chambers view. Atrial situs solitus, levocardia with levoapex, and atrioventricular discordance. Abbreviations: RA-right atrium; RV-right ventricle; LA-left atrium; LV-left ventricle; Ao-Aorta; PA-pulmonary artery; RB-right branch; lb-left branch; (b) MRI sagittal view. Systemic right morphological ventricular connection with the aorta; (c) MRI volumetric reconstruction. Morphological left ventricle connection with the pulmonary artery.

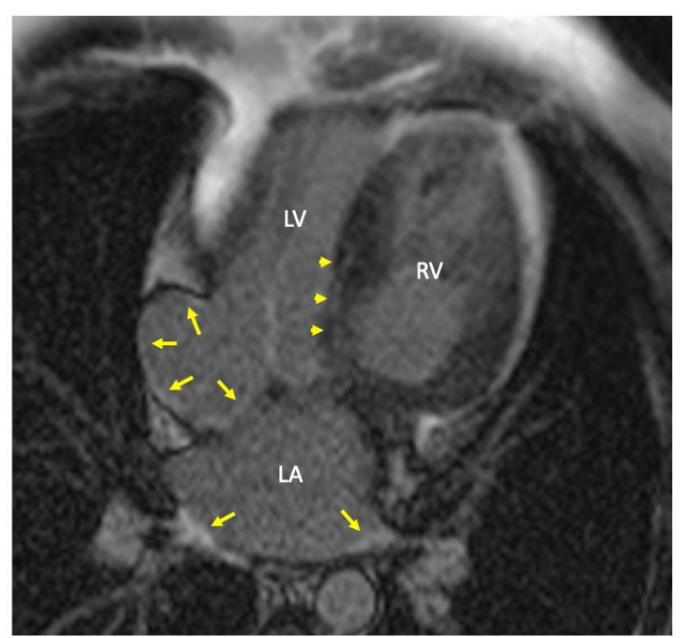

Figure 3. Atrial fibrosis: MRI 4 chambers view, postcontrast late enhancement sequence, hyperintensity in both atria (yellow arrows) and in the intramyocardial ventricular septum (yellow head arrows). Abbreviations as before.

The case was presented to the cardiothoracic surgery department and an anatomical correction with tricuspid and mitral valvular replacement was recommended, and a VVI pacemaker placement, due to the conduction anomalies. The patient refused surgical management but accepted medical treatment. Right and left cardiac catheterization was performed, which also demonstrated mitral and tricuspid regurgitation, but without signs of pulmonary hypertension despite the pulmonary artery dilation previously reported on MRI.

After pacemaker placement, the patient has an improvement of clinical symptoms. His medication on discharge was aspirin $100 \mathrm{mg}$ daily, captopril 25 mg twice daily, and cephalexin $500 \mathrm{mg}$ three times a day for seven days. A follow-up appointment was arranged but the patient refused further management due to improvement of symptoms after pacemaker placement. 


\section{Discussion}

CCTGA is a rare congenital heart disease, found in about 1 in 33,000 newborns and accounting for $0.05 \%$ of all heart congenital disorders [7], that has been frequently associated with supraventricular arrhythmias-mainly paroxystic supraventricular tachyarrhythmia and atrial fibrillation, atrioventricular blocks due to left atrium dilation and in some cases, it has even been directly associated with ventricular tachycardia [8] [9].

Compared to other cases of CCTGA in adults, our patient had a milder presentation. Many patients have been reported in the medical literature at this age with signs and symptoms of congestive heart failure, which is their main complaint. It is important to do a close follow-up to identify early signs of decompensation and treat them appropriately [2].

The decision to make a surgical intervention must be evaluated deeply depending on a case-to-case basis and the paraclinical findings. In previous studies, it has been found that patients who were surgically corrected before advanced stages of heart failure had better clinical outcomes. In a similar way, patients with CCTGA and conduction abnormalities who had pacemaker placement had fewer complications associated with congestive heart failure decompensations. An important difference in our patient is the lack of other findings besides the blocked atrial fibrillation. In future studies, better indicators of surgical correction are needed for patients who do not present any clinical findings [5] [10].

It can be argued that atrial fibrillation would be related to biatrial dilation in patients with CCTGA. In our patient, probably the main cause of his atrial fibrillation was the presence of atrial fibrosis demonstrated on MRI. Recent studies have shown that atrial fibrosis is the leading cause of atrial fibrillation rather than atrial dilation [11] [12].

Incomplete right bundle branch block (RBBB) has occasionally been found in these patients because they develop right ventricular dilation and right heart failure [2] [10]. Nevertheless, it has not been described in patients without right heart failure, such as ours.

Despite the syncope probably related to arrhythmia and radiological findings of increased pulmonary flow (right-to-left shunt), it never presented any clinical signs of right heart failure. This leads us to believe that RBBB was not in fact due to heart failure, as previously reported [2] [10], but was most likely caused by the presence of fibrosis described in the interventricular septum.

The lack of symptoms turned out to be counterproductive, as the patient felt well and refused surgical management.

\section{Conclusion}

Cardiac arrhythmias are the leading cause of death from CCTGA, primarily ventricular tachycardia, and atrial fibrillation. $\mathrm{RBBB}$ is a very rare and previously unreported presentation in this congenital heart disease. This, added to the fact that our patient was diagnosed late without symptoms of heart failure, makes it 
an atypical case of CCTGA.

\section{Conflicts of Interest}

The authors declare no conflicts of interest regarding the publication of this paper.

\section{References}

[1] Connelly, M., Liu, P., Williams, W., Webb, G., Robertson, P. and McLaughlin, P. (1996) Congenitally Corrected Transposition of the Great Arteries in the Adult: Functional Status and Complications. Journals of the American College of Cardiology, 27, 1238-1243. https://doi.org/10.1016/0735-1097(95)00567-6

[2] Friedberg, D. and Nadas, A. (1970) Clinical Profile of Patients with Congenital Corrected Transposition of the Great Arteries. New England Journal of Medicine, 282, 1053-1059. https://doi.org/10.1056/NEJM197005072821901

[3] Baruteau, A., Abrams, D., Ho, S., Thambo, J., McLeod, C. and Shah, M. (2017) Cardiac Conduction System in Congenitally Corrected Transposition of the Great Arteries and Its Clinical Relevance. Journal of the American Heart Association, 6, e007759. https://doi.org/10.1161/JAHA.117.007759

[4] Zhang, L., Liu, H., Wan, Q. and Han, X. (2019) Ventricular Fibrillation in Congenitally Corrected Transposition of Great Arteries Treated with Pacing: A Case Report. European Heart Journal-Case Reports, 3, 1-6. https://doi.org/10.1093/ehjcr/ytz199

[5] Agarwal, A., Samad, F., Kalvin, L., Bush, M. and Tajik, A. (2017) A Great Imitator in Adult Cardiology Practice: Congenitally Corrected Transposition of the Great Arteries. Congenital Heart Disease, 12, 143-152. https://doi.org/10.1111/chd.12453

[6] Krishnan, K., Avramovitch, N., Kim, M. and Trohman, R. (2005) Cardiac Resynchronization Therapy: A Potential Option for Congenitally Corrected Transposition of the Great Vessels. Heart Rhythm, 2, S254-S255. https://doi.org/10.1016/j.hrthm.2005.02.797

[7] Wallis, G., Debich-Spicer, D. and Anderson, R. (2011) Congenitally Corrected Transposition. Orphanet Journal of Rare Diseases, 6, 22. https://doi.org/10.1186/1750-1172-6-22

[8] Tseng, W., Huang, C., Chiu, S., Lu, C., Wang, J., Lin, M., et al. (2021) Long-Term Outcomes of Arrhythmia and Distinct Electrophysiological Features in Congenitally Corrected Transposition of the Great Arteries in an Asian Cohort. American Heart Journal, 231, 73-81. https://doi.org/10.1016/j.ahj.2020.10.057

[9] Almahmeed, W., Haykowski, M., Boone, J., Kavanagh-Gray, D. and Macdonald, H.I. (1996) Congenitally Corrected Transposition of the Great Arteries and Exercise-Induced Ventricular Tachycardia. Canadian Journal of Cardiology, 12, 526-528.

[10] Hornung, T., Bernard, E., Celermajer, D., Jaeggi, E., Howman-Giles, R., Chard, R., et al. (1999) Right Ventricular Dysfunction in Congenitally Corrected Transposition of the Great Arteries. American Journal of Cardiology, 84, 1116-1119. https://doi.org/10.1016/S0002-9149(99)00516-0

[11] Goette, A. (2002) Determinants and Consequences of Atrial Fibrosis in Patients Undergoing Open Heart Surgery. Cardiovascular Research, 54, 390-396. https://doi.org/10.1016/S0008-6363(02)00251-1

[12] Gal, P. and Marrouche, N. (2015) Magnetic Resonance Imaging of Atrial Fibrosis: Redefining Atrial Fibrillation to a Syndrome. European Heart Journal, 38, 14-19. https://doi.org/10.1093/eurheartj/ehv514 